Pharmaco-
logical
inhibition
of IRAK4
signalling
suppresses
inflammatory
and fibrotic
markers

FIBROSIS

\title{
Pericyte MyD88 and IRAK4 signalling in fibrosis
}

New data suggest that signalling of MyD88 and its downstream effector IRAK4 in pericytes is an important driver of inflammation and fibrogenesis in the injured kidney. Jeremy Duffield and colleagues also show that pharmacological inhibition of IRAK4 signalling suppresses inflammatory and fibrotic markers in kidney tissue of mice following ischaemiareperfusion injury (IRI), suggesting that this pathway might represent a therapeutic avenue for the treatment of renal fibrosis.

Previous work by Duffield and others has shown that pericytes are a source of myofibroblasts in renal fibrogenesis. To investigate the mechanism by which pericytes respond to injury, Duffield and colleagues performed transcriptional analyses, identifying upregulation of a large number of innate immunityrelated pathways in pericytes in response to injury. Specifically, the Toll-like receptors, TLR2 and TLR4, and the adaptor molecule, MyD88, controlled secretion of cytokines, chemokines and growth factors by pericytes in response to damage-associated molecular patterns (DAMPs) released from damaged kidneys. Activation of pericytes by DAMPs led to activation of the NLRP3 inflammasome and secretion of IL- $1 \beta$ and IL- 18 , while IL- $1 \beta$ led to further pericyte activation.

Pericytes lacking Myd88 or Tlr2/4 were unable to migrate or express fibroblast markers in response to TGF- $\beta$, implicating these pathways in the fibrogenic process. The researchers also showed that mice with pericyte-specific deletion of Myd88 had significantly reduced levels of inflammation in response to IRI compared to levels in wildtype mice. Moreover, a new small-molecule inhibitor of IRAK4 led to reduced fibrosis, inflammation, and improved renal outcomes.

Susan J. Allison

ORIGINAL ARTICLE Leaf, I. A. et al. Pericyte MyD88 and IRAK4 control inflammatory and fibrotic responses to tissue injury.J. Clin. Invest. http://dx.doi.org/10.1172/JCl87532 (2016) 